OPEN

SUBJECT AREAS:

TWO-DIMENSIONAL

MATERIALS

APPLIED PHYSICS

Received

23 October 2014

Accepted

22 January 2015

Published

17 February 2015

Correspondence and requests for materials should be addressed to

A.J.H.M.

(mcgaughey@cmu.

edu)

\section{Strongly anisotropic in-plane thermal transport in single-layer black phosphorene}

\author{
Ankit Jain \& Alan J. H. McGaughey
}

Department of Mechanical Engineering, Carnegie Mellon University, Pittsburgh, PA 15213.

Using first principles calculations, we predict the thermal conductivity of the two-dimensional materials black phosphorene and blue phosphorene. Black phosphorene has an unprecedented thermal conductivity anisotropy ratio of three, with predicted values of $110 \mathrm{~W} / \mathrm{m}-\mathrm{K}$ and $36 \mathrm{~W} / \mathrm{m}-\mathrm{K}$ along its armchair and zigzag directions at a temperature of $300 \mathrm{~K}$. For blue phosphorene, which is isotropic with a zigzag structure, the predicted value is $78 \mathrm{~W} / \mathrm{m}-\mathrm{K}$. The two allotropes show strikingly different thermal conductivity accumulation, with phonons of mean free paths between $10 \mathrm{~nm}$ and $1 \mu \mathrm{m}$ dominating in black phosphorene, while a much narrower band of mean free paths $(50-200 \mathrm{~nm})$ dominate in blue phosphorene. Black phosphorene shows intriguing potential for strain-tuning of its thermal conductivity tensor.

wo-dimensional (2D) materials (e.g., graphene, $\mathrm{MoS}_{2}$, silicene) are a focus of intense research because of their rich physics and potential for integration into next-generation electronic and energy conversion devices ${ }^{1-4}$. As opposed to their bulk counterparts, the optical, electronic, mechanical, and thermal properties of 2D materials can be easily tailored through the application of external strain, by introducing defects, or by stacking multiple layers of the same or different 2D materials. For example, the thermal conductivity of freelysuspended single-layer graphene is reduced from $3000-5000 \mathrm{~W} / \mathrm{m}-\mathrm{K}$ to $600 \mathrm{~W} / \mathrm{m}-\mathrm{K}$ by depositing it on amorphous $\mathrm{SiO}_{2}{ }^{5}$.

Recently, a new and promising 2D semiconductor, black phosphorene, was fabricated by exfoliating a few layers from bulk black phosphorus ${ }^{6,7}$. Similar to graphene, black phosphorene has a honeycomb-like structure, but it is non-planar [Fig. 1(a)]. Single-layer black phosphorene is a direct-gap semiconductor with a predicted band gap of $2 \mathrm{eV}^{8}$. The band gap decreases with an increasing number of layers and is $0.3 \mathrm{eV}$ for the bulk phase. Performance characteristics similar to or better than other 2D materials have been achieved for a black phosphorene-based transistor ${ }^{7}$. What distinguishes black phosphorene from other 2D materials is its anisotropic structure, which leads to direction-dependent optical and electronic properties that vary by as much as $50 \%{ }^{8,9}$.

Our objective is to study the lattice thermal conductivity of single-layer black phosphorene, for which there is no existing experimental or theoretical data available. Ong et al. investigated the effect of strain on the ballistic thermal conductance of black phosphorene using non-equilibrium Green's functions based on harmonic lattice dynamics calculations ${ }^{10}$. Our aim is to predict the thermal conductivity of black phosphorene by including anharmonic phonon-phonon scattering. Thermal transport characterization is important for application in most devices, where large electrical currents can lead to Joule heating, non-radiative recombination, and potentially high operating temperatures. We find that the in-plane thermal transport in black phosphorene is strongly anisotropic, with thermal conductivity varying by a factor of three over the two orthogonal directions. While strong anisotropy in thermal conductivity is observed for van der Waals layered materials when comparing the in-plane and cross-plane directions (e.g., two orders of magnitude in graphite ${ }^{11}$ ), no other covalently-bonded 2D or 3D materials show the in-plane anisotropy we predict for black phosphorene.

We also investigate the thermal transport in blue phosphorene, another single-layered allotrope of phosphorus [Fig. 1(b)], which was recently predicted to be nearly as stable as black phosphorene ${ }^{12}$. First principles calculations predict a band gap in blue phosphorene in excess of $2 \mathrm{eV}^{12}$, but unlike black phosphorene, blue phosphorene is isotropic.

\section{Methods}

Thermal transport in semiconductors like black phosphorene and blue phosphorene is dominated by atomic vibrations whose energy is quantized as phonons. The phonon contribution to thermal conductivity in the $l$ direction can be calculated using a solution to the 


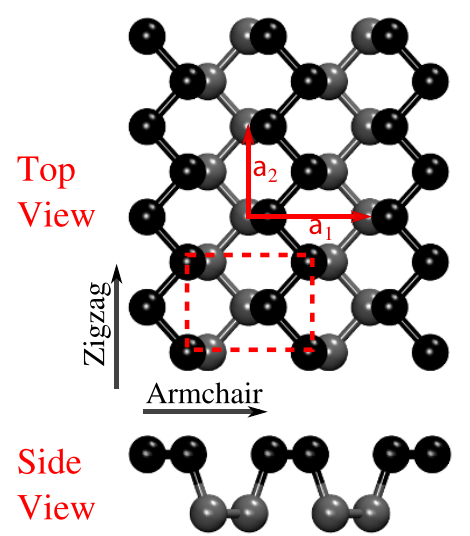

(b)

Blue

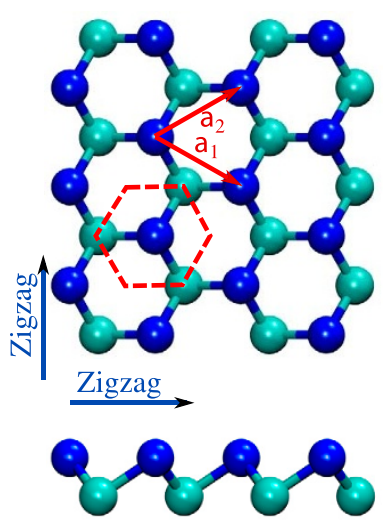

Figure $1 \mid$ Crystal structure of (a) black phosphorene and (b) blue phosphorene. Atoms in different planes are denoted by different colors and the unit cell and the in-plane lattice vectors are shown in the top views. Black phosphorene has a four-atom unit cell with an armchair structure along $\mathbf{a}_{1}$ and a zigzag structure along $\mathbf{a}_{2}$. Blue phosphorene has a two-atom unit cell with the zigzag structure along $\mathbf{a}_{1}$ and $\mathbf{a}_{2}$. The side views show the armchair and zigzag structures. The lattice constants are $\left|\mathbf{a}_{1}\right|=4.43 \AA$ and $\left|\mathbf{a}_{2}\right|=3.28 \AA$ for black phosphorene, and $\left|\mathbf{a}_{1}\right|=\left|\mathbf{a}_{2}\right|=3.15 \AA$ for blue phosphorene.

Boltzmann transport equation (BTE) that uses the Fourier law, giving ${ }^{13,14}$

$$
k_{l}=\sum_{i} c_{p h, i} v_{g, l, i}^{2} \tau_{l, i}
$$

The summation in Eq. (1) is over all the phonon modes in the first Brillouin zone. The mode index, $i \equiv\left(\begin{array}{c}\boldsymbol{\kappa} \\ v\end{array}\right)$, enumerates phonon wave vector, $\boldsymbol{\kappa}$, and polarization, $v$. On the right-hand side of Eq. (1), $c_{p h, i}$ is the volumetric specific heat, $v_{g, l, i}$ is the $l$ component of the phonon group velocity vector $\mathbf{v}_{g, i}$, and $\tau_{l, i}$ is the phonon lifetime. The phonon mean free path is $\left|\mathbf{v}_{g, i}\right| \tau_{l, i}$. The specific heat is calculated using BoseEinstein statistics. The group velocity vector is related to the mode frequency, $\omega_{i} \equiv \omega_{v}^{\kappa}$, as $\mathbf{v}_{g, i}=\partial \omega_{v}^{\boldsymbol{\kappa}} / \partial \boldsymbol{\kappa}$. The phonon frequencies are obtained by diagonalizing the dynamical matrix and the phonon lifetimes are obtained using an iterative (full) solution of the linearized BTE for phonons ${ }^{15}$. The phonon-phonon scattering rates are obtained by considering three-phonon interactions in the Fermi golden rule. At a temperature of $300 \mathrm{~K}$, the electronic contribution to the thermal conductivity of black phosphorene is predicted to be less than $3 \mathrm{~W} / \mathrm{m}-\mathrm{K}$ for a typical carrier concentration of $10^{12} \mathrm{~cm}^{-2} 16$.

In our thermal conductivity calculations, the only required inputs are harmonic and anharmonic force constants, which are obtained from density functional theory (DFT) and density functional perturbation theory (DFPT) calculations. We use a scalar relativistic pseudopotential generated using the projector augmented-wave method as implemented in the plane wave-based quantum-chemistry package

Quantum Espresso ${ }^{17}$. The plane wave energy cutoff is $50 \mathrm{Ry}$. To remove inter-layer interactions due to the periodicity of the computational cell, we use a vacuum of $30 \AA$ for black phosphorene and $17 \AA$ for blue phosphorene. For black phosphorene, the harmonic force constants are calculated on phonon and electronic wave-vector grids of $14 \times 12 \times 1$. The cubic force constants are obtained by finite differencing of Hellmann-Feynman forces on a 144-atom supercell with a Gamma-point electronic wave-vector grid. For blue phosphorene, the electronic and phonon wave-vector grids for the harmonic force constants are $10 \times 10 \times 1$ and the Hellmann-Feynman forces are obtained using a 128-atom supercell with a Gamma-point electronic wave-vector grid. For the thermal conductivity calculation, the phonon wave vector grid is $50 \times 50$ $\times 1$ for both allotropes. Translational invariance (i.e., the acoustic sum rule) for the cubic force constants is enforced using the Lagrangian approach presented by $\mathrm{Li}$ et al. ${ }^{18}$. We note that the thermal conductivities are converged within $20 \%(10 \%)$ for black (blue) phosphorene for the above choice of parameters. Further details regarding these choices are provided in the supplementary information (SI).

\section{Results}

Phonon Dispersion. The phonon dispersion in the high-symmetry directions of the first Brillouin zone for black phosphorene and blue phosphorene are plotted in Figs. 2(a) and 2(b) and closely match those reported by Zhu et al. ${ }^{12}$. Black(blue) phosphorene has a four(two)-atom unit cell, resulting in twelve(six) dispersion branches. The maximum phonon frequency is similar in both
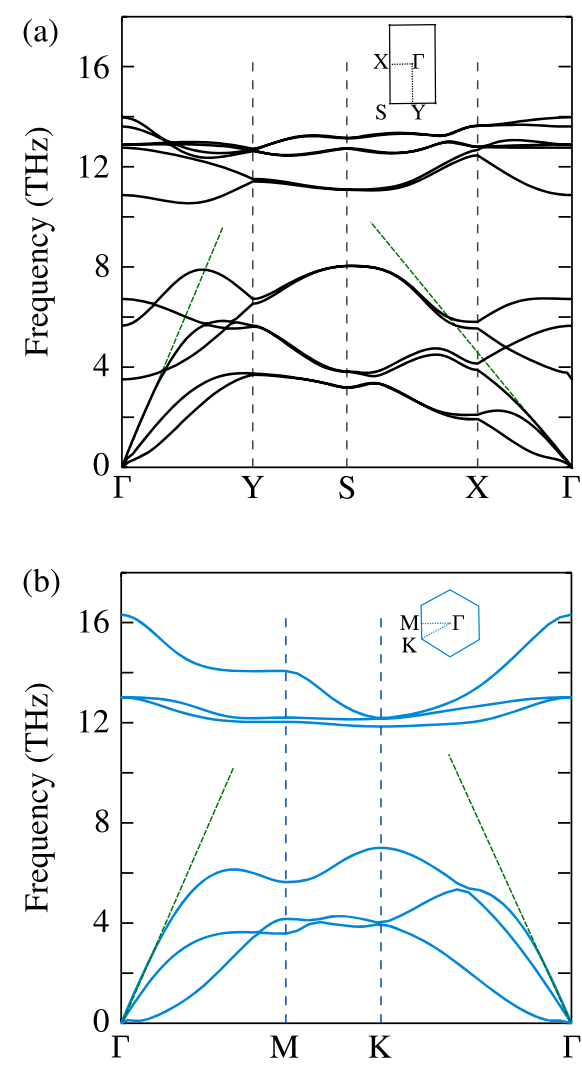

Figure $2 \mid$ Phonon dispersion in the high symmetry directions for (a) black phosphorene and (b) blue phosphorene. The slope of the longitudinal acoustic phonon branches at the Gamma point (shown as dashed straight lines), which represents the sound velocity, is anisotropic for black phosphorene and isotropic for blue phosphorene.

allotropes (14.0 THz in black and $16.3 \mathrm{THz}$ in blue), but the phonon band gap in blue phosphorene $(4.9 \mathrm{THz})$ is almost double that in black phosphorene $(2.5 \mathrm{THz})$. The longitudinal acoustic phonon group velocity close to the $\Gamma$ point (i.e., the sound speed), is $7,733 \mathrm{~m} / \mathrm{s}$ in the $\Gamma-\mathrm{Y}$ (armchair) direction and 4,168 m/s in the $\Gamma-\mathrm{X}$ (zigzag) direction for black phosphorene, an indication of anisotropic phonon transport. For blue phosphorene, the sound velocity is $8,287 \mathrm{~m} / \mathrm{s}$ in both the $\Gamma-\mathrm{M}$ and $\Gamma-\mathrm{K}$ directions.

Thermal Conductivity. The thermal conductivities of black phosphorene and blue phosphorene for temperatures between 200 and $500 \mathrm{~K}$ are plotted in Fig. 3(a). For black phosphorene, thermal transport is anisotropic and we plot the thermal conductivity in both the armchair and zigzag directions [see Fig. 1(a)]. Predicting the thermal conductivity of a $2 \mathrm{D}$ material requires specification of the layer thickness. We choose the bulk layer separation, which is $5.25 \AA$ for black phosphorus ${ }^{19}$ and $5.63 \AA$ for blue phosphorus ${ }^{12}$. As the thermal conductivity scales linearly with the layer thickness, the values reported here can be easily modified for other choices.

Thermal conductivity decreases with increasing temperature, as expected for a phonon-dominated crystalline material. At a temperature of $300 \mathrm{~K}$, the predicted thermal conductivities are $110 \mathrm{~W} / \mathrm{m}-\mathrm{K}$ (zigzag) and $36 \mathrm{~W} / \mathrm{m}-\mathrm{K}$ (armchair) for black phosphorene and $78 \mathrm{~W} / \mathrm{m}-\mathrm{K}$ for blue phosphorene. For black phosphorene, the thermal conductivity in the zigzag direction is three times higher than that in the armchair direction. This anisotropy could be useful in the design of heat channeling in micro- and nano-devices. We attribute this anisotropy in thermal conductivity to the anisotropic phonon dispersion, which leads to direction-dependent group velocities [Fig. 2(a)]. Based simply on the zone-center longitudinal acoustic group velocities, Eqn. 1 predicts a thermal conductivity anisotropy of 

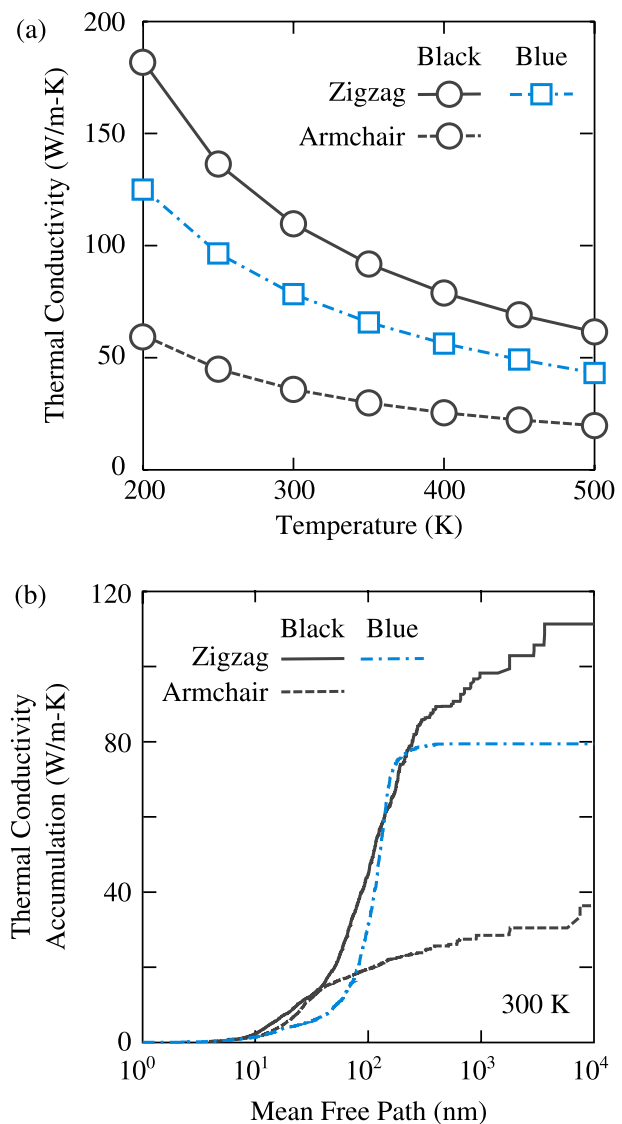

Figure $3 \mid$ (a) Temperature-dependent thermal conductivity of black phosphorene and blue phosphorene. The thermal conductivities are obtained using an iterative solution of the linearized BTE. The predictions (symbols) are connected using lines to guide the eye. (b) Thermal conductivity accumulation functions for black phosphorene and blue phosphorene at a temperature of $300 \mathrm{~K}$.

3.5, which is comparable to that from the full calculation. For blue phosphorene, the thermal conductivity is isotropic and is up to 1.8 times lower than the zigzag direction thermal conductivity of black phosphorene.

Our thermal conductivities are obtained using an iterative solution of the linearized BTE. For single-layer graphene, Lindsay et al. showed that the commonly used relaxation time approximation (RTA) of the BTE under-predicts the thermal conductivity by more than a factor of five at a temperature of $300 \mathrm{~K}^{22}$. We find that the RTA under-predicts the thermal conductivity by up to a factor of 1.3 for black phosphorene and 2.0 for blue phosphorene at a temperature of $300 \mathrm{~K}$ (see SI).

The thermal conductivity of black phosphorene has recently also been predicted by other researchers. Zhu et al. used the RTA to predict values of $84 \mathrm{~W} / \mathrm{m}-\mathrm{K}(24 \mathrm{~W} / \mathrm{m}-\mathrm{K})$ in the zigzag (armchair) direction $^{20}$. These values closely match our RTA predictions of $81 \mathrm{~W} / \mathrm{m}-\mathrm{K}(30 \mathrm{~W} / \mathrm{m}-\mathrm{K})$ (see SI). Qin et al. predict black phosphorene thermal conductivities a factor of three lower than our values ${ }^{21}$. This difference may be due to (i) their use of the RTA, (ii) their choice of the cubic force constant cutoff, and/or (iii) the implementation of a translational invariance constraint on the third-order force constants. Our predictions of the effects of these factors on thermal conductivity are presented in the SI.

In Fig. 3(b), we plot the thermal conductivity accumulation functions for black phosphorene and blue phosphorene at a temperature of $300 \mathrm{~K}$. The thermal conductivity accumulation function describes the contribution of different mean free path phonons towards the total thermal conductivity of a material ${ }^{23}$. Phonons with mean free paths spanning over two orders of magnitude $(10 \mathrm{~nm}$ to $1 \mu \mathrm{m})$ contribute towards the thermal conductivity in black phosphorene. For blue phosphorene, however, the accumulation function closely resembles a step function, with the major contribution coming from phonons with mean free paths between 50 and $200 \mathrm{~nm}$. This steep thermal conductivity accumulation in blue phosphorene is similar to that in silicene (which also has buckled hexagonal structure), where phonons with mean free path between 5 and $20 \mathrm{~nm}$ contribute more than $80 \%$ to the thermal conductivity ${ }^{24}$. The thermal conductivity accumulation functions indicate that, unlike in graphene, where thermal conductivity is predicted to increase with sample sizes even greater than $10 \mu \mathrm{m}$, changing the sample size beyond $10 \mu \mathrm{m}$ will have a minimal effect on the thermal conductivity of either phosphorene allotrope at a temperature $300 \mathrm{~K}$.

As mentioned above, the structure of blue phosphorene is similar to that of silicene. Blue phosphorene, however, has a thermal conductivity of $78 \mathrm{~W} / \mathrm{m}-\mathrm{K}$ at a temperature of $300 \mathrm{~K}$, which is more than eight times higher than that predicted for silicene $(9.4 \mathrm{~W} / \mathrm{m}$ $\mathrm{K})^{24}$. This higher thermal conductivity of blue phosphorene is the result of a larger sound velocity and the larger frequency gap in its phonon dispersion [Fig. 2(b)], which reduces the number of threephonon scattering processes that can satisfy the energy conservation selection rule ${ }^{25-28}$. The zigzag direction thermal conductivities of black and blue phosphorene are comparable in magnitude to that predicted for a $10 \mu \mathrm{m} \mathrm{MoS}$ sample prediction at a temperature of $300 \mathrm{~K}(108 \mathrm{~W} / \mathrm{m}-\mathrm{K})^{29}$.

At a temperature of $300 \mathrm{~K}$, Lindsay et al. ${ }^{30}$ predicted the thermal conductivity of graphene to be $3,600 \mathrm{~W} / \mathrm{m}-\mathrm{K}$, which is more than 30 times higher than the thermal conductivity of either black phosphorene or blue phosphorene. We believe that the lower thermal conductivity of the phosphorene allotropes is due to their: (i) smaller sound velocities $\left(4,000-8,000 \mathrm{~m} / \mathrm{s}\right.$ compared to $21,300 \mathrm{~m} / \mathrm{s}$ in graphene $\left.{ }^{31}\right)$, (ii) lower Debye temperatures [500 K (see SI) compared to 2,300 K for graphene $\mathrm{e}^{32}$, resulting in higher phonon-phonon scattering rates as more phonon modes are active at a given temperature, and (iii) non-planar structure, which breaks the out-of-plane symmetry. This out-of-plane symmetry exists in graphene and restricts the participation of odd numbers of ZA phonons in phonon-phonon scattering events ${ }^{22}$. As a result, the predicted contribution of ZA phonons to thermal conductivity in the phosphorene allotropes varies from 12-

Table 1 | Contribution of different phonon modes branches [longitudinal acoustic (LA), transverse acoustic (TA), out-of-plane acoustic (ZA), and all optical] towards the total thermal conductivity in black phosphorene, blue phosphorene, $\operatorname{MoS}_{2}\left(10 \mu \mathrm{m}\right.$ sample $\left.{ }^{29}\right)$, and graphene $\left(10 \mu \mathrm{m}\right.$ sample $\left.^{30}\right)$ at a temperature of $300 \mathrm{~K}$

\begin{tabular}{lcccr} 
Material & \multicolumn{1}{c}{ Total thermal conductivity (W/m-K) } & LA (\%) & TA (\%) & ZA (\%) \\
\hline Black phosphorene (zigzag) & 110 & 32 & 22 & 31 \\
Black phosphorene (armchair) & 36 & 28 & 33 & 12 \\
Blue phosphorene & 78 & 26 & 27 & 44 \\
MoS $_{2}^{29}$ & 108 & 28 & 24 & 34 \\
Graphene $^{30}$ & 3600 & 8 & 15 & 76 \\
\hline
\end{tabular}


(a)
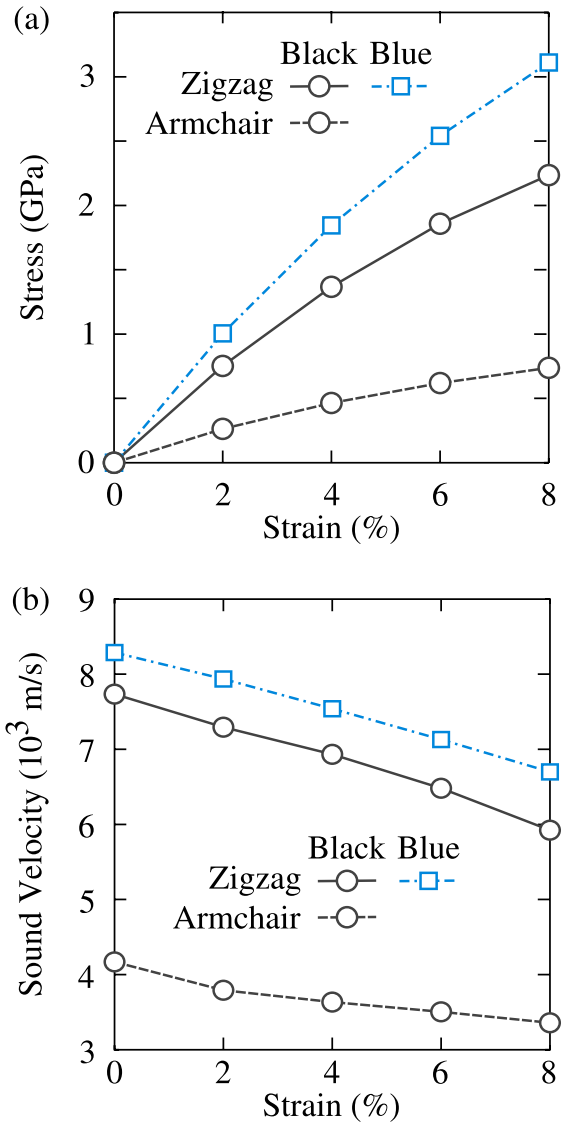

Figure $4 \mid$ (a) Stress and (b) sound velocity in black phosphorene and blue phosphorene under bi-axial strain. The predicted values (symbols) are connected using lines to guide the eye.

$44 \%$ as compared to $76 \%$ in graphene at a temperature of $300 \mathrm{~K}^{30}$, as presented in Table 1 . We note that the thermal conductivity contributions of the different acoustic phonon branches in black phosphorene and blue phosphorene are similar to those predicted for $\mathrm{MoS}_{2}{ }^{29}$, which also has a non-planar structure. More information is provided in the $\mathrm{SI}$.

The thermal conductivity of freely-suspended single-layer graphene at a temperature of $300 \mathrm{~K}$ is reduced from $3000-5000 \mathrm{~W} /$ $\mathrm{m}-\mathrm{K}$ to $600 \mathrm{~W} / \mathrm{m}-\mathrm{K}$ when it is deposited on amorphous $\mathrm{SiO}_{2}{ }^{5}$. This more than a factor of five reduction in the thermal conductivity is due to the increased scattering of ZA phonons in supported graphene. The results presented in this work are for suspended phosphorene. As can be seen in Table 1, the contribution of ZA phonons is $31 \%(12 \%)$ in the zigzag (armchair) direction in black phosphorene and $44 \%$ in blue phosphorene.
We expect a comparable decrease in the thermal conductivity of supported phosphorene samples.

Strain Tuning of Thermal Conductivity. We now consider the possibility of strain-tuning the thermal conductivity of black phosphorene and blue phosphorene. In Fig. 4(a), we plot the stresses in both allotropes when they are subjected to a bi-axial tensile strain. For black phosphorene, the stress is anisotropic and is three times lower in the armchair direction compared to the zigzag direction. For blue phosphorene, which has a zigzag structure, the stress is isotropic and is 1.5 times larger than the stress in the zigzag direction of black phosphorene.

As can be seen from Eqn. 1, thermal conductivity scales as the square of the phonon group velocities. We plot the strain-dependence of the sound velocities in Fig. 4(b) for black phosphorene and blue phosphorene under the bi-axial strain conditions. The sound velocities are maximum at zero strain for both materials and decrease with increasing strain. Using this reduction as a guide, we estimate that the thermal conductivities of both allotropes may decrease by a factor of 1.7 at a strain of $8 \%$. Similar calculations for uni-axial strains suggest the possibility for strain-tailoring the thermal conductivity anisotropy in black phosphorene. For example, the estimated anisotropy in the thermal conductivity of black phosphorene is a factor of 4.6 (2.2) for uni-axial strain of $6 \%$ along the armchair (zigzag) direction (see SI).

Anisotropy. To the best of our knowledge, no other 2D material displays anisotropic in-plane thermal transport. Furthermore, the thermal conductivity anisotropy in black phosphorene is striking when compared to that found in layered, wurtzite, and orthorhombic three-dimensional crystal structures, as compiled in Table 2. We quantify anisotropy, $r$, by the ratio of the maximum and minimum direction-dependent thermal conductivities. The maximum $r$ is in the layered structures graphite and hexagonal boron nitride. These layers are weakly bonded through van der Waals interactions compared to the strong covalent bonds within the layers, which results in poor interlayer heat transport. Of the remaining materials in Table 2, which are all covalently bonded, the maximum anisotropy is for our prediction for black phosphorene, which is twice as large as the next highest value.

\section{Summary}

We predicted the thermal conductivity of black phosphorene and blue phosphorene using first-principles-driven lattice dynamics calculations and a full (iterative) solution of the BTE. We found a factor of three anisotropy in the thermal conductivity of black phosphorene, which could potentially be tuned up to 4.6 using strain. At a temperature of $300 \mathrm{~K}$, the predicted thermal conductivities of both phosphorene allotropes are larger than that of silicene, similar to that of $\mathrm{MoS}_{2}$, and are up to two order of magnitude smaller than that of graphene.

Table 2 | Anisotropy in thermal conductivity for selected materials with layered, wurtzite, and orthorhombic crystal structure at a temperature of $300 \mathrm{~K}$. 'exp' and 'pred' in the first column denote experimental measurements and simulation predictions

\begin{tabular}{lccr} 
Material & $k_{\max }(\mathrm{W} / \mathrm{m}-\mathrm{K})$ & $k_{\min }(\mathrm{W} / \mathrm{m}-\mathrm{K})$ & $r$ \\
\hline Graphite $\left(\exp ^{33}\right)$ & $(1000-2000)$ & 6 & 2 \\
h-BN $\left(\exp ^{33}\right)$ & $(200-300)$ & $\mathbf{3 6}$ & $\sim 300$ \\
Black Phosphorene & $\mathbf{1 1 0}$ & 0.46 & $\mathbf{3}$ \\
$\mathrm{SnSe}\left(\exp ^{34}\right)$ & 0.70 & 385 & 1.52 \\
$\mathrm{GaN}\left(\right.$ pred $\left.^{14}\right)$ & 401 & 303 & 1.04 \\
$\mathrm{AlN}\left(\right.$ pred $\left.^{14}\right)$ & 322 & 2.68 & 1.06 \\
$\mathrm{NdFeO}_{3}\left(\right.$ pred $\left.^{35}\right)$ & 3.01 & 5.72 & 1.12 \\
$\mathrm{NdAlO}_{3}\left(\right.$ pred $\left.^{35}\right)$ & 6.61 & 1.16 & \\
\hline
\end{tabular}


1. Novoselov, K. S. et al. Electric field effect in atomically thin carbon films. Science 306, 666-669 (2004)

2. Zhang, Y., Tan, Y.-W., Stormer, H. L. \& Philip, K. Experimental observation of the quantum Hall effect and Berry's phase in graphene. Nature 438, 201-204 (2005).

3. Bolotin, K. et al. Ultrahigh electron mobility in suspended graphene. Solid State Commun. 146, 351-355 (2008).

4. Ramakrishna Matte, H. S. S. et al. $\mathrm{MoS}_{2}$ and $\mathrm{WS}_{2}$ analogues of graphene. Angew. Chem. Int. Edit. 49, 4059-4062 (2010).

5. Seol, J. H. et al. Two-dimensional phonon transport in supported graphene. Science 328, 213-216 (2010).

6. Liu, H. et al. Phosphorene: An unexplored 2d semiconductor with a high hole mobility. ACS Nano 8, 4033-4041 (2014).

7. Li, L. et al. Black phosphorus field-effect transistors. Nat. Nano. 9, 372-377 (2014).

8. Tran, V., Soklaski, R., Liang, Y. \& Yang, L. Layer-controlled band gap and anisotropic excitons in few-layer black phosphorus. Phys. Rev. B 89, 235319 (2014).

9. Fei, R. \& Yang, L. Strain-engineering the anisotropic electrical conductance of few-layer black phosphorus. Nano Lett. 14, 2884-2889 (2014).

10. Ong, Z.-Y., Cai, Y., Zhang, G. \& Zhang, Y.-W. Strong thermal transport anisotropy and strain modulation in single-layer phosphorene. J. Phys. Chem. C 118, 25272-25277 (2014).

11. Balandin, A. A. et al. Superior thermal conductivity of single-layer graphene. Nano Lett. 8, 902-907 (2008).

12. Zhu, Z. \& Tománek, D. Semiconducting layered blue phosphorus: A computational study. Phys. Rev. Lett. 112, 176802 (2014).

13. Reissland, J. A. The Physics of Phonons (John Wiley and Sons Ltd, New York, 1973).

14. Lindsay, L., Broido, D. A. \& Reinecke, T. L. Ab initio thermal transport in compound semiconductors. Phys. Rev. B 87, 165201 (2013).

15. Omini, M. \& Sparavigna, A. Beyond the isotropic assumption in the theory of thermal conductivity. Phys. Rev. B 53, 9064-9073 (1996).

16. Lv, H. Y., Lu, W. J., Shao, D. F. \& Sun, Y. P. Enhanced thermoelectric performance of phosphorene by strain-induced band convergence. Phys. Rev. B 90, 085433 (2014).

17. Giannozzi, P. et al. Quantum espresso: a modular and open-source software project for quantum simulations of materials. J. Phys.-Condens. Mat. 21, 395502 (2009).

18. Li, W., Lindsay, L., Broido, D. A., Stewart, D. A. \& Mingo, N. Thermal conductivity of bulk and nanowire $\mathrm{Mg}_{2} \mathrm{Si}_{x} \mathrm{Sn}_{1-x}$ alloys from first principles. Phys. Rev. B 86, 174307 (2012).

19. Zachariasen, W. The atomic arrangement in glass. J. Am. Chem. Soc. $\mathbf{5 4}$, 3841-3851 (1932)

20. Zhu, L., Zhang, G. \& Li, B. Coexistence of size-dependent and size-independent thermal conductivities in phosphorene. Phys. Rev. B 90, 214302 (2014).

21. Qin, G. et al. Anisotropic intrinsic lattice thermal conductivity of phosphorene from first principles. Phys. Chem. Chem. Phys. 17, 4854 (2014).

22. Lindsay, L., Broido, D. A. \& Mingo, N. Flexural phonons and thermal transport in graphene. Phys. Rev. B 82, 115427 (2010).

23. Dames, C. \& Chen, G. Thermal conductivity of nanostructured thermoelectric materials. Thermoelectrics Handbook: Macro to Nano. Rowe, D. M. (ed.). (CRC Press, Boca Raton, 2005).
24. Xie, H., Hu, M. \& Bao, H. Thermal conductivity of silicene from first-principles. Appl. Phys. Lett. 104, 131906 (2014).

25. Jain, A. \& McGaughey, A. J. H. Thermal conductivity of compound semiconductors: Interplay of mass density and acoustic-optical phonon frequency gap. J. Appl. Phys. 116, 073503 (2014)

26. Lindsay, L., Broido, D. A. \& Reinecke, T. L. First-principles determination of ultrahigh thermal conductivity of boron arsenide: A competitor for diamond? Phys. Rev. Lett. 111, 025901 (2013).

27. Broido, D. A., Lindsay, L. \& Reinecke, T. L. Ab initio study of the unusual thermal transport properties of boron arsenide and related materials. Phys. Rev. B. 88, 214303 (2013).

28. Lindsay, L., Broido, D. A. \& Reinecke, T. L. Thermal conductivity and large isotope effect in gan from first principles. Phys. Rev. Lett. 109, 095901 (2012).

29. Li, W., Carrete, J. \& Mingo, N. Thermal conductivity and phonon linewidths of monolayer $\mathrm{MoS}_{2}$ from first principles. Appl. Phys. Lett. 103, 253103 (2013).

30. Lindsay, L. et al. Phonon thermal transport in strained and unstrained graphene from first principles. Phys. Rev. B 89, 155426 (2014).

31. Maultzsch, J., Reich, S., Thomsen, C., Requardt, H. \& Ordejón, P. Phonon dispersion in graphite. Phys. Rev. Lett. 92, 075501 (2004).

32. Efetov, D. K. \& Kim, P. Controlling electron-phonon interactions in graphene at ultrahigh carrier densities. Phys. Rev. Lett. 105, 256805 (2010).

33. Shindé, S. L. \& Goela, J. High thermal conductivity materials (Springer, Berlin, 2006).

34. Zhao, L.-D. et al. Ultralow thermal conductivity and high thermoelectric figure of merit in SnSe crystals. Nature 508, 373-377 (2014).

35. Steele, B., Burns, A., Chernatynskiy, A., Grimes, R. \& Phillpot, S. Anisotropic thermal properties in orthorhombic perovskites. J. Mater. Sci. 45, 168-176 (2010).

\section{Author contributions}

A.J. performed the calculations. A.J. and A.J.H.M. analyzed the data. A.J. wrote the manuscript. A.J.H.M. reviewed and edited the manuscript.

\section{Additional information}

Supplementary information accompanies this paper at http://www.nature.com/ scientificreports

Competing financial interests: The authors declare no competing financial interests.

How to cite this article: Jain, A. \& McGaughey, A.J.H. Strongly anisotropic in-plane thermal transport in single-layer black phosphorene. Sci. Rep. 5, 8501; DOI:10.1038 srep08501 (2015).

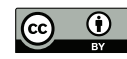

This work is licensed under a Creative Commons Attribution 4.0 International License. The images or other third party material in this article are included in the article's Creative Commons license, unless indicated otherwise in the credit line; if the material is not included under the Creative Commons license, users will need to obtain permission from the license holder in order to reproduce the material. To view a copy of this license, visit http://creativecommons.org/licenses/by/4.0/ 